\title{
Essais
}

Revue interdisciplinaire d'Humanités

Résister entre les lignes

\section{Cántico espiritual de Jean de la Croix et de Blas de Otero. Étude comparative}

\section{Violeta Bereghici}

\section{(2) OpenEdition \\ 1 Journals}

Édition électronique

URL : http://journals.openedition.org/essais/4703

DOI : $10.4000 /$ essais.4703

ISSN : 2276-0970

Éditeur

École doctorale Montaigne Humanités

\section{Édition imprimée}

Date de publication : 15 juillet 2016

Pagination : 158-172

ISBN : 978-2-9544269-8-3

ISSN : 2417-4211

\section{Référence électronique}

Violeta Bereghici, "Cántico espiritual de Jean de la Croix et de Blas de Otero. Étude comparative », Essais [En ligne], 9 | 2016, mis en ligne le 23 octobre 2020, consulté le 29 octobre 2020. URL : http:// journals.openedition.org/essais/4703; DOI : https://doi.org/10.4000/essais.4703 


\title{
Cántico espiritual de Jean de la Croix et de Blas de Otero. Étude comparative
}

\section{Violeta Bereghici}

\author{
Ni por gracia y hermosura \\ yo nunca me perderé, \\ sino por un no sé qué \\ que se halla por ventura. \\ Jean de la Croix $^{1}$
}

Notre objet d'étude portera sur deux recueils de poésie couronnés par le même titre - Cántico espiritual -, de deux poètes majeurs de la poésie espagnole : Jean de la Croix (1542-1591) et Blas de Otero (1916-1979).

Liées à jamais à la spiritualité, la pensée et l'œuvre de Jean de la Croix ${ }^{2}$ éclatent au grand jour au $\mathrm{XX}^{\mathrm{e}}$ siècle grâce à des études critiques très fructueuses aussi bien en Espagne que hors de ses frontières et notamment en France. Tout au long de son existence, Jean de la Croix s'est soumis à la volonté divine et

1 San Juan de la Cruz, Glosa a lo divino, in Cántico espiritual y poesía completa, éd. Francisco Rico, vol. 44, Barcelona, 2002, p. 243.

2 "Jean de la Croix, Juan de Yepes de son vrai nom, est né en 1542 à Fontiveros en Vielle Castille. Après avoir exercé en tant qu'infirmier à l'hôpital de Medina del Campo, il décide d'entrer dans un couvent de carmes de cette même localité. Il étudie la théologie à l'université de Salamanque, l'université la plus renommée d'Europe à l'époque. En 1568, il commence la réforme des carmes et prend le nom de Jean de la Croix. Dix ans plus tard les carmes non réformés le font enlever de nuit et l'enferment dans un cachot à Tolède. Pendant neuf mois et dans des conditions de vie extrêmement difficiles, il compose mentalement puis par écrit des poésies dont le Cántico Espiritual contenant des poèmes et des gloses qui retracent le cheminement de l'âme vers l'union avec Dieu. Prieur, recteur de collège, vicaire provincial, Jean de la Croix a consacré toute sa vie à la diffusion du message divin aussi bien auprès des âmes consacrées qu'auprès des laïcs. Après une campagne de calomnies, Jean de la Croix meurt dans la solitude en 1591 et au-delà, la persécution se poursuivra contre ses écrits qui ne seront publiés que vingt-sept ans après sa mort. Ce retard sera exploité par les plagiaires et explique la diffusion de nombreuses copies manuscrites, mais aussi les déformations et les mutilations de son œuvre. Il faudra attendre le $\mathrm{XX}^{\mathrm{e}}$ siècle pour avoir une édition critique et des textes authentiques ». Bord André, Jean de la Croix en France, éd. Beauchesne, Paris 1993, p. 16-17. 
a immortalisé son expérience mystique à l'aide d'une écriture poétique d'une remarquable élégance où il retrace le chemin que doit parcourir l'âme pour rencontrer Dieu. En effet, la destination finale vers laquelle le grand poète mystique veut conduire l'homme est contenue dans le titre La Montée du Mont Carmel: il s'agit d'arriver au sommet de la lumière et de la révélation divines où seront possibles l'union et l'amour de l'âme avec Dieu.

Au sein de la production littéraire de Jean de la Croix ${ }^{3}$, on distingue le bloc strophique composé de trente-neuf liras intitulé Cántico espiritual, un véritable joyau poétique qui ne pouvait pas laisser indifférents nombre de disciples parmi lesquels se trouve le poète moderne Blas de Otero. Avec d'autres poètes tels que Gabriel Celaya, José Hierro, Eugenio de Nora, Ramón de Girasol, Leopoldo de Luis, Blas de Otero fait partie de la génération dite « de l'aprèsguerre » et puise son inspiration dans la réalité immédiate, dans la situation réelle du pays ${ }^{4}$. Consciente de sa responsabilité et de son devoir, cette génération de poètes proclame un idéal commun : sortir l'Espagne du marasme par le recours à un langage poétique direct, nu, dépourvu de tout symbole superflu. Blas de Otero s'inscrit parfaitement dans cet idéal d'existence et d'engagement par la précision et la rigueur de l'expression poétique ainsi que par la dimension culturelle et historique qu'alimentent toutes ses compositions.

Afin d'éloigner l'homme des atrocités de la guerre et des méfaits de la dictature, Blas de Otero le place au centre de ses préoccupations poétiques et le conduit vers un bonheur que seule l'expérience spirituelle peut procurer. Pour ce faire, il va puiser son inspiration dans le Cántico espiritual de Jean de la Croix. En effet, quatre siècles plus tard, et plus exactement en 1942, à l'occasion de l'anniversaire des 400 ans de la naissance de Jean de la Croix, Blas de Otero présente son récital de poésie intitulé Cántico espiritual. Ce titre représente le début d'une activité poétique féconde du poète espagnol modernes.

3 On fait généralement une distinction entre les œuvres mineures et les œuvres majeures de Jean de la Croix. Les œuvres mineures se composent de poèmes improvisés avec des mètres différents, des conseils spirituels, des précautions (cautelas), des lettres, des maximes. Parmi les œuvres majeures, on distingue La Nuit (huit strophes commentées deux fois), La montée du Mont Carmel qui correspond à l'aspect actif des nuits (nuit du sens : Livre I ; nuit de l'esprit : Livre II ; purification de la mémoire et de la volonté : Livre III), La Nuit obscure correspond à l'aspect passif de la nuit (nuit passive du sens : Livre I ; nuit passive de l'esprit : Livre II).

4 Blas de Otero, Parler clair, (En castellano), traduit de l'espagnol par Claude Couffon, éditeur Pierre Seghers, Paris, 1959, p. 8.

5 Deux livres, Ángel fieramente humano, 1950 (Ange sauvagement humain) et Redoble de conciencia, 1951 (Rappel de conscience) sont le vrai départ d'une œuvre brève et dense. Ancia reprend sous un titre de synthèse les deux premiers recueils complétés de quelques nouveaux poèmes où se font entendre les échos déchirants d'un Quevedo, d'un Unamuno et des réminiscences des psaumes bibliques. Le regard se détourne peu à peu d'un Ciel implacablement vide et se pose sur la terre habitée représentant la seconde étape de l'itinéraire poétique de Blas de Otero avec trois recueils: Pido la paz y la palabra, 1955 (Je demande la paix et la parole), En castellano, 1959 (Parler clair) ; Que trata de España, 1964 (Qui traite de l'Espagne). Poésie sociale, 
La coïncidence du titre Cántico espiritual constitue le point de départ de notre projet de lecture. Placé dans une perspective comparative, il portera sur la spécificité du dialogue inter et intrapoétique grâce à ce moteur dynamique d'écriture qu'est le signifiant : de quelle manière et par quels moyens discursifs le signifiant, par sa prégnance et par son autonomie, par sa résistance et par sa liberté, s'érige-t-il en automatisme associatif ${ }^{6}$ poétique ? Comment d'une époque à l'autre, d'un poète à l'autre, d'un style à l'autre, le signifiant demeure-t-il immuable, y compris dans l'activité inconsciente ? Telles sont les questions auxquelles nous essaierons de répondre au fil de ces pages.

\section{L'avènement du signifiant onomastique}

Pour intituler son premier recueil de poésie, Blas de Otero choisit effectivement ce titre déjà existant dans la poésie espagnole : le Cántico espiritual du grand poète espagnol, intellectuel et théologien du XVI ${ }^{e}$ siècle, Jean de la Croix. Un tel choix invite avant tout à une lecture des poèmes du poète mystique et, dès la deuxième strophe, Canción $\mathbf{2}$ de son Cántico espiritual, une première surprise se présente au lecteur :

ainsi a-t-on qualifié le lyrisme qui prend en compte la réalité des hommes et leur combat. Les derniers recueils de Blas de Otero - Hojas de Madrid, 1968 (Feuilles de Madrid), Mientras, 1970 (Cependant), Historias fingidas y verdaderas, 1970 (Histoires feintes et véritables) - témoignent d'un certain retour à des thèmes plus personnels et d'une libération de la forme : rythmes plus amples, métrique plus souple, images neuves, mots insolites, retour à un certain hermétisme ". Bernard Sesé, "Otero Blas de (1916-1979) », Encyclopedia Universalis (en ligne) : http://www. universalis-edu.com/encyclopedie/blas-de-otero.

6 Nous définissons l'automatisme comme étant le résultat d'une période de raisonnement et d'acquisition que le cerveau a conservés à l'aide de la mémoire et de la répétition. Plus on répète l'action d'une voie, y compris par simple imagination, plus cette voie se renforce et plus le geste auquel elle correspond va devenir automatique. Considéré donc comme le résultat de processus complexes d'ordre psychique, cognitif, linguistique, l'automatisme s'apparente à une instance bien constituée, stable, disponible et rapide permettant aux opérateurs psychocognitifs de faire des économies d'énergie et de conserver leurs potentialités pour des activités supérieures plus soutenues. Même si les automatismes peuvent entraîner des comportements figés, ils sont indispensables à notre fonctionnement. Sinon, nous serions obligés d'apprendre chaque jour les mêmes choses. Sans automatismes, nous ne pourrions pas posséder le langage. Ils constituent un " trésor " solide susceptible d'être sollicité et mobilisé à tout moment. Qualifier l'automatisme d' " associatif ", c'est lui attribuer des propriétés associatives, c'est-àdire des capacités à s'associer dans des combinaisons multiples. En effet, l'automatisme s'inscrit dans une dynamique associative permettant de faire " du nouveau avec du vieux ». Ce sont les liens et les combinaisons qui rendent les automatismes vivants et aptes à s'inscrire dans des significations toujours nouvelles et surprenantes. Elles ne sont pas toujours explicites, notamment dans le langage poétique où l'automatisme apparaît dans des combinaisons inattendues du fait qu'il est substituable et interchangeable, bien que toujours organisé au sein d'un réseau associatif sans lequel l'automatisme serait un électron libre dépourvu de toute existence et de toute cohabitation heureuse. 
2

Pastores, los que fueredes

allá por las majadas al otero,

si por ventura vieredes

aquel que yo más quiero,

decidle que adolezco, peno y muero. ${ }^{7}$

Il s'agit de l'apparition du signifiant " otero " avec minuscule, anagrammatisé aussi sous l'écorce du premier mot de la strophe "pasTOREs » et de la locution " pOR vEnTura» (v. 3), anagrammes qui, pour être parfaites, exigent le dédoublement de la voyelle " $\mathbf{0}$ ». Le même signifiant apparaît également dans la Canción 12 :

12

Apártalos, Amado,

Que voy de vuelo.

Vuélvete, paloma,

que el ciervo vulnerado

por el otero asoma

al aire de tu vuelo y fresco toma. ${ }^{8}$

La deuxième occurrence du signifiant "otero " se trouve également anagrammatisée deux fois dans le dernier vers « al aiRe de Tu vuElO y fREscO TOma ", la première anagramme exigeant le dédoublement de la voyelle « $\mathbf{0}$ " pour être totale.

Cette lecture littérale linéaire et anagrammatique permet de constater la présence importante du signifiant " otero » : une occurrence linéaire par strophe doublée de deux occurrences anagrammatisées basées sur le jeu de la symétrie avec toute la signification et avec toute la symbolique que connotent les différents chiffres, pairs, impairs, additions : Canción $2+$ Canción $12=(1+2)+$ $(1+2)=3+3=2+4$. La symbolique du nombre accompagne le poète et son œuvre, car « les chiffres ont été de tout temps aussi support de rêve, de fantasme, de spéculation métaphysique, matériau de la littérature, sondes de l'avenir incertain ou du moins du désir de prédire. Autant que les mots, ou presque, ils ont été les outils du poète... ", comme l'explique Georges Ifrah. . L'unité du chiffre 1, par exemple, renvoie à des instances singulières telles que Dieu, l'âme, le poète, la poésie, le titre, et, son amplification jusqu'au double (2) instaure une relation dichotomique : Esposo/Esposa, Amado/Amado, âme/dieu, divino/ humano, ciervo/paloma, etc. Ce dualisme structure notre projet de lecture et d'analyse qui porte sur deux recueils, deux poètes, deux époques, deux visions, les voix d'interlocution yo/tú, deux strophes, deux mètres (hendécasyllabe et

7 San Juan de la Cruz, Cántico espiritual y poesía completa, éd. Francisco Rico, vol. 44, Barcelona, 2002, p. 8.

8 Ibid., p. 18.

9 Ifrah Georges, Histoire universelle des chiffres, Robert Laffont, Paris, 1994, p. 18. 
heptasyllabe), deux occurrences linéaires et deux multipliées par deux $(2 \times 2)$ occurrences anagrammatisées du signifiant " otero ".

Bien que le signifiant " otero " n'entretienne aucun lien, du moins à première vue, avec le signifiant onomastique "Otero ", il convient d'examiner sa signification dans le contexte de ces deux strophes de Jean de la Croix.

Dans la Canción 2 dont le lexique employé rappelle la poésie pastorale de Garcilaso et de Juan del Encina, l'âme, nommée allégoriquement Esposa/ Amada, demande aux bergers d'être ses messagers et de communiquer à Dieu (Esposo/Amado), s'ils l'aperçoivent quelque part dans les altitudes lointaines (" allá por las majadas al otero »), l'intensité de son amour et l'immensité de sa souffrance causées par Son absence. La convocation de tiers, " pastores ", ${ }^{10}$ ouvre la voie à une recherche itinérante spirituelle qui va d'un lieu statique et fermé "majadas " (" lugares donde se albergan los pastores y se recoge el ganado de noche ") vers un lieu haut et ouvert où se trouve Dieu : " el otero ", terme que Covarrubias définit comme "lugares altos que descubren campo", Francisco de Rosal ajoute "otear : mirar de alto " et "otero : de donde se otea "; le Diccionario de Autoridades présente " otero " comme " el lomo que hace la tierra, elevándose en los llanos, de forma que sobresale a los demás ». ${ }^{11}$

L'ouverture et la verticalité de l'espace rendues par le signifiant "otero " vont de pair avec l'indétermination, la virtualité et la vacuité de ce même espace emphatisé par le déictique " allá ", participant ainsi à la création d'une sensation de perspective et d'un espace plus personnel, plus subjectif, apparenté à l'espace psychique.

La deuxième occurrence du signifiant "otero " dans la Canción 12 marque une rupture significative : pour la première fois apparaît celui qui est tant recherché et désiré (" el ciervo vulnerado/por el otero asoma ") grâce à l'emploi du signifiant « asomar ", terme qui selon le Diccionario de Autoridades, signifie " empezar a mostrarse alguna cosa ». Le Diccionario etimológico de Francisco de Rosal fait dériver "asomar " de "somo, que es lo alto, del Lat. Summo, y así asomar parecer por lo alto ", coïncidant avec la définition de Covarubias, " parecer por lo alto » et le Diccionario crítico etimológico castellano e hispánico (DECH) de Corominas qui donne l'étymon, SUMMUS, "el más alto ", " comienza a aparecer por lo alto del otero ". ${ }^{12} \mathrm{Si}$ " asomar " dérive de "somo ", "summo " et si l'équivalent français de " otero " est "sommet "

10 «Pastor » est un des noms du Christ (Pastor de pastores) chez Fray Luis de León. "Pastores » ou " apacentadores " sont également des intermédiaires plus abstraits comme l'affection et le désir permettant à l'âme de communiquer avec Dieu. " Pastores " peut également représenter les anges qui non seulement servent de messagers dans les deux sens, de l'âme vers Dieu et de Dieu vers l'âme, mais protègent l'âme contre les forces du Mal.

11 Cruz San Juan de la, Notas complementarias, in Cántico espiritual y poesía completa, éd. Francisco Rico, vol. 44, Barcelona, 2002, p. 565.

12 Ibid., p. 574. 
("Là-haut par vos abris au sommet ", "Sur le sommet se penche ») ${ }^{13}$, il y a, dans cette compréhension transversale et bilingue, comme une redondance, un trop-plein de signification qui fait que cette idée de hauteur, de sommet est non seulement l'espace "platonique " de l'amour divin et humain, mais aussi l'espace poétique. Ce haut lieu de la poésie où la fonction poétique renforce et met en évidence l'importance, l'organisation et l'habillage phonique du signifiant représente l'espace idéal susceptible de véhiculer un nouveau signifié superposé au signifié explicite.

L'apparition de Dieu dans ce lieu de contemplation et de communication " otero " transforme le chant solitaire de l'âme en dialogue comme figure de l'incarnation du Verbe, favorisé par le mouvement descendant de Dieu (" por el otero asoma ") et par le mouvement ascendant de l'âme (" que voy de vuelo »), ce qui rend imminente la rencontre ou plus exactement ici, la révélation divine. Celle-ci implique aussi le dialogue métrique qui s'établit entre l'hendécasyllabe et l'heptasyllabe au sein de la composition strophique de cinq vers qu'est la lira $^{14}$. Cette rencontre de la versification est favorisée aussi par la vocation qu’à la langue espagnole de ne pas séparer "l'identité sémantique des mots de leur rythme, de l'accent dit tonique, qui met fortement en relief l'une des syllabes et laisse les autres en semi-sourdine, ce qui fait que la prosodie des mots castillans est déjà une mesure et une métrique ", écrit Nadine Ly. ${ }^{15}$

De nombreuses réflexions critiques montrent que le terme «otero » dans la poésie de Jean de la Croix jouit d'un emploi polysémique et métaphorique. ${ }^{16}$ Cette perspective polysémique et métaphorique du terme " otero "

13 Ly Nadine, Anthologie bilingue de la poésie espagnole, Bibliothèque de la Pléiade, Éditions Gallimard, Paris, 1995.

14 «Introduite en Espagne par Garcilaso au milieu du XVI siècle, la lira connaît immédiatement une diffusion exceptionnelle. Profane chez Garcilaso, elle se spiritualise avec Fray Luis de León et se "divinise » avec saint Jean de la Croix. Dans les Odes de Fray Luis, l'équilibre et la tension entre les deux mètres (l'hendécasyllabe et l'heptasyllabe) servent à l'expression d'un chant supérieur fait de plénitudes et de ruptures, de conflits entre l'harmonie éternelle et le désarroi humain. Chez saint Jean, le dialogue amoureux de deux mètres exalte la double quête de l'Époux et de l'Épouse et l'ardeur unanime de leur rencontre sensuelle et spirituelle. Il ne lui sera plus jamais accordé, dans la poésie espagnole, de place aussi prestigieuse. Le regain qu'elle connaît au XVIII ${ }^{e}$ siècle, la renvoie à sa fonction originelle d'imitation des mètres classiques. Mais elle renaitra, au XXI siècle, comme élément d'embellissement d'autres formes strophiques : sous la plume d'Antonio Machado, ses heptasyllabes allégeront les quatrains d'hendécasyllabes, et l'adjectif alirado définit les compositions auxquelles elle prête ses exceptionnelles qualités poétiques ». Ly Nadine, Anthologie bilingue de la poésie espagnole, Bibliothèque de la Pléiade, Éditions Gallimard, Paris, 1995, p. XXX.

15 Ibid., p. IX.

16 «Parker (1985 : 113) encuentra que el otero constituye una metáfora simbólica, que Mancho (1993a : 172) relaciona con otros símbolos ascensionales sanjuanistas, favorecedores de la dilatación y profundidad de la mirada, del dominio visual e intelectivo. [...] A. Egidi (1991a : 84) considera las majadas y el otero como loci mnemotécnicos que guardan imágenes, unas estáticas y otras dinámicas, referidas a un recuerdo que enciende, desde el presente, el deseo hacia el 
demande à être élargie et enrichie par l'analyse sémiotique, cette dernière étudiant la question de la matrice même du signe, de son organisation et de son fonctionnement, de ses mutations et de ses limites. Si, en effet, le signifiant " otero " n'exige aucune manipulation vocale, il demande toutefois une association mentale pouvant aller jusqu'à une sorte de "rêverie associative ", impliquant aussi bien le poète « onomaturge » que le lecteur.

"Il y a une part totalement anonyme qui erre dans les noms ", telle est l'affirmation de Goethe que Nadine Ly $^{17}$ reprend et place au cœur de sa remarquable réflexion sur l'anonymat des noms et des choses. Cette part totalement anonyme qui erre sans doute aussi dans le signifiant " otero " semble sortir de l'anonymat et prendre place dans le lexique mental du lecteur moderne, notamment dans celui de l'hispaniste, pour parvenir à désigner un référent nouveau. Dans la perspective de notre analyse, cette part totalement anonyme peut être comprise aussi comme une part totalement inconsciente, ce qui n'est pas sans faire référence à la notion freudienne de "résidus archaïques ", appelée par Jung " archétypes " ou "images primordiales " ${ }^{18}$. À l'instar de la phylogenèse de l'espèce, la vie que donne l'écriture au signifiant n'est en fait qu'une répétition abrégée, un héritage archaïque, et comme dans le rêve, dans le signifiant se perpétue une part ancestrale qui échappe à la compréhension et à la conscience. C'est du moins la lecture que nous faisons ici de l'emploi de ce signifiant. Il importe toutefois de prendre conscience qu'il s'agit là d'un signifiant facile à anagrammatiser et il est permis de se demander si l'inconscient joue un rôle véritable.

En effet, à la lumière de ces quelques réflexions, l'image acoustique et graphique du signifiant " otero " induit, par connotation sémiotique, le nom propre du poète Blas de Otero. Ce sommet, ce « otero » de Jean de la Croix, lieu à la fois de révélation divine et d'inspiration poétique, est aussi le lieu de la révélation onomastique où le signifiant " otero ", par procédé de démotivation du signe et par glissement vers un autre référent extralinguistique, s'élève au statut de signifiant onomastique "Otero ». Grâce à ce pur hasard, à ce " por ventura " cher à Jean de la Croix, cet « inconscient textuel à vocation visionnaire » nous permet, en poussant l'analyse littérale à son plus haut degré de discernement, de le considérer comme une prédiction inconsciente du poète mystique fort révélatrice de ce signifiant prégnant près « d'accoucher » à la fois d'un même (signifiant) et d'un autre (référent/signature). Quatre siècles plus tôt, Jean de la Croix apparaît inconsciemment comme un visionnaire et fait

futuro. Icaza destaca la ambigüedad de este símbolo secundario, que designa a una localización en que se halla el amado y donde se produce la amorosa contemplación ». Cruz San Juan de la, Cántico espiritual y poesía completa, éd. Francisco Rico, vol. 44, Barcelona, 2002, p. 470.

17 Ly Nadine, Nommer, Littéralité 4, PUB, 2002, p. 13-56.

18 Jung C. G., Essai de l'exploration de l'inconscient, Folio, Denoël, 2013, p. 117-118. 
figurer dans son œuvre poétique le nom de celui qui sera le chef de file de la génération des poètes d'après-guerre et pour qui le langage poétique doit être direct, spontané, dénudé et dépouillé de toute superficialité. Cette inspiration divine et poétique qui descend du haut de la poésie de Jean de la Croix, Blas de Otero la placera, par le recours au procédé associatif poétique, au centre de son premier recueil, Cántico espiritual.

Si le grand poète mystique occupe une place de visionnaire, il en va autrement pour le poète moderne qui connaissait parfaitement l'œuvre de Jean de la Croix, dont l'hommage commence par un choix délibéré et conscient au moment d'intituler son premier recueil Cántico espiritual. Ce titre n'est en aucun cas une simple citation, une réminiscence ancienne ou un hommage apparent fait au poète mystique. Comme nous l'avons dit précédemment, le Cántico espiritual est le récital de poésie présenté par Blas de Otero en 1942, exactement au moment de l'anniversaire des 400 ans de la naissance de Jean de la Croix né à Fontiveros (Moraña) en 1542, le signifiant " otero » se trouve anagrammatisé aussi dans le toponyme "fOnTivEROs ».

Une manifestation moins inconsciente est à noter dans un des derniers poèmes du Cántico espiritual de Blas de Otero, intitulé $A$ que sí, qui convoque le nom propre du poète mystique - San Juan de la Cruz en espagnol - au moyen du signifiant " Cruz » :

Y si en una Cruz murió,

no sería, creo yo, a que no. ${ }^{19}$

para abrirnos más la herida,

A que no, cuando muramos,

pensaremos en esa luz

viene de esta misma Cruz

que se alzó por ti, por mí.

A que no... A que sí... ${ }^{20}$

Le signifiant "Cruz " désigne dans ces vers le lieu de la crucifixion et les verbes de mouvement "abrirnos " et "se alzó " contribuent à ce que ce lieu soit connoté sémantiquement par la même ouverture et la même verticalité dont nous avons qualifié l'espace défini par « otero ». Bien qu'il s'agisse de la croix sur laquelle a été crucifié le Christ, le signifiant "Cruz " avec majuscule retient toute l'attention du lecteur : cette double occurrence du signifiant "Cruz » représente l'image symétrique en miroir de la double occurrence du signifiant « otero", schématisée comme suit :

19 Blas de Otero, Obra completa, Edición de Sabina de la Cruz con la colaboración de Mario Hernández, Galaxia Gutemberg, p. 123.

20 Ibid., p. 124. 


\begin{tabular}{|c|c|}
\hline $\begin{array}{l}\text { Jean de la Croix } \\
\text { Cántico espiritual }\end{array}$ & $\begin{array}{c}\text { Blas de Otero } \\
\text { Cántico espiritual }\end{array}$ \\
\hline otero (Canción 2, p. 8) & Cruz (A que sí, verso 4, p. 123) \\
\hline otero (Canción 12, p. 18) & Cruz (A que sí, verso 32, p. 124) \\
\hline
\end{tabular}

Les signifiants " otero, Cruz » entrent en résonance associative avec la locution prépositionnelle " por ti, por mí » formant ainsi un chiasme qui, en dehors de l'implication de l'instance divine, permet d'associer à un autre niveau de lecture les deux voix lyriques tú Cruz/yo Otero, réunies dans un dialogue poétique qui plaide en faveur d'une cause commune - divine et poétique - emphatisée par des verbes impliquant la première personne du pluriel : " para abrirnos ", " cuando muramos ", " pensaremos ». Et c'est à partir de ce dialogue que Blas de Otero débutera le déploiement d'associations à des niveaux discursifs variés (lexical, poétique, thématique), ce qui lui permettra d'exprimer poétiquement sa pensée religieuse certes, mais surtout sa vision de l'écriture poétique par l'élévation d'une série d'associations littéraires à un haut degré d'automaticité, les transformant tout au long de son écriture en automatismes associatifs poétiques. Ce sont précisément les automatismes associatifs poétiques qui, grâce à la perpétuation et au pouvoir évocateur du signifiant, rendent possible cette belle rencontre poétique, comme nous le verrons dans les lignes qui suivent.

\section{Suprématie du signifiant}

Associant les deux voix d'interlocution, le dialogue interpoétique accompagne et soutient une autre forme de dialogue : le dialogue intrapoétique, basé sur l'omniprésence des mêmes signifiants dans les deux recueils en commençant par une série de titres qui coïncident ou se rapprochent analogiquement :

\begin{tabular}{|c|c|}
\hline Jean de la Croix & Blas de Otero \\
\hline $\begin{array}{c}\text { Cántico espiritual } \\
\text { Canciones 1-39 }\end{array}$ & Cántico espiritual \\
I, II, III
\end{tabular}

Or, ce dialogue intra poétique ne se limite pas aux seuls titres : il s'inscrit dans une heureuse continuité et se construit avec les mêmes mots, vers, strophes, poèmes. Voici quelques exemples présentés sous forme de tableaux : 


\begin{tabular}{|l|l|}
\hline \multicolumn{1}{|c|}{ Jean de la Croix } & \multicolumn{1}{c|}{ Blas de Otero } \\
\hline ciervo (p. 7, 18, 35) & ciervo (p. 107) \\
\hline pastores (p. 8) & pastores (p. 123) \\
\hline flores (p. 9,10, 37, 32) & flores (p. 123) \\
\hline paloma (p. 18) & paloma (p. 100) \\
\hline espesuras (p. 10, 41) & espesuras (p. 107) \\
\hline ojos (p. 28, 29) & ojos [p. 99, 100, 101, 103 (cinq occurrences)] \\
\hline aire (p. 44) & aire (p. 103) \\
\hline agua (p. 45) & agua (p. 101) \\
\hline montes (p. 41) & montes (p. 107) \\
\hline collado (p. 41) & Collados (p. 107) \\
\hline otero (p. 8, 18) & otear (p. 109) \\
\hline subidas (p. 42) & altura (p. 97, 109, 112, 119) \\
\hline hermosura (p. 11, 30, 41) & hermosura (p. 110, 113) \\
\hline $\begin{array}{l}\text { por ventura (p. 2, 8, 243, 244, 245, etc., } \\
\text { et neuf occurrences dans « Glosa a lo divino ») }\end{array}$ & por ventura (p. 113) \\
\hline amenas liras (p. 36) & $\begin{array}{l}\text { lira de paz (p. 114) } \\
\text { íntima lira (p. 118) }\end{array}$ \\
\hline
\end{tabular}

Loin d'être complet, ce répertoire du lexique utilisé par les deux poètes à des fins poétiques bien précises est doublé d'une multitude de similitudes et d'analogies entre les vers et les strophes : 


\begin{tabular}{|c|c|}
\hline Jean de la Croix & Blas de Otero \\
\hline Salí tras ti clamando, y eras ido. (p. 1) & $\begin{array}{l}\text { Gimo y clamo hacia Ti como un pecado } \\
\text { (p. 100) } \\
\text { Sońé..., y te habías ido (p. 109) }\end{array}$ \\
\hline decidle que adolezco, peno y muero (p. 12) & $\begin{array}{l}\text { Que en ella me adolezco, peno y muero (p. 11) } \\
\text { Me adolezco de vientos precursores (p. 99) }\end{array}$ \\
\hline $\begin{array}{l}\text { vestido los dejó de hermosura (p. 5) } \\
\text { que gracia y hermosura en mi dejaste (p. 24) } \\
\text { y vámonos a ver en tu hermosura (p. 335) }\end{array}$ & $\begin{array}{l}\text { se adolece oteando la Hermosura (p. 109) } \\
\text { dolidos de hermosura (p. 110) } \\
\text { con ella, la Hermosura (p. 113) }\end{array}$ \\
\hline $\begin{array}{l}\text { la noche sosegada (p. 20) } \\
\text { la soledad sonora (p. 20) }\end{array}$ & $\begin{array}{l}\text { la noche sosegada y robadora } \\
\text { la soledad sonora (p. 112) }\end{array}$ \\
\hline las ínsulas extrañas (p. 13) & Todo el mundo, Señor, es una isla (p. 104) \\
\hline $\begin{array}{l}18 \\
\text { Allí me dio su pecho, } \\
\text { allí me enseñó ciencia muy sabrosa, } \\
\text { y yo le di de hecho } \\
\text { a mí, sin dejar cosa; } \\
\text { allí le prometí de ser su esposa. (p. 24) }\end{array}$ & $\begin{array}{l}\text { Por ella yo quisiera } \\
\text { dormirme entre los brazos del Esposo } \\
\text { muriendo de manera } \\
\text { tan alta, y silencioso, } \\
\text { que abriérame este pecho que reboso. (p. 114) }\end{array}$ \\
\hline $\begin{array}{l}\text { Cuando tú me mirabas, } \\
\text { su gracia en mí tus ojos imprimían, } \\
\text { por eso me adamabas, } \\
\text { y en eso merecían } \\
\text { los míos adorar lo que en ti vían. (veían) } \\
\text { (p. 29) }\end{array}$ & $\begin{array}{l}\text { Adiós. Hay unos ojos que nos miran } \\
\text { Y nos clavan ; sentimos su presencia } \\
\text { En el costado, como un alfiler } \\
\text { En tierna mariposa... Y eran bellos } \\
\text { Eran amados al sonar las cinco, } \\
\text { Eran los ojos que quisimos ver. (p. 101) }\end{array}$ \\
\hline
\end{tabular}

Une lecture attentive de ces quelques exemples et du discours poétique des trente-neuf chansons, excepté la glose en prose qui accompagne chaque lira, permet de constater que Jean de la Croix utilise un lexique qui n'a rien d'un vocabulaire religieux, mais qui forme un réseau associatif caractéristique de la poésie courtoise et pastorale avec toutes ses composantes : l'amour (amado/a, esposo/a, adamar, adorar, hermosura, gracia, etc.), la faune (ciervo, paloma), la flore (flores, espesura), l'air (aire, vuelo), le relief (otero, montes, collado, altura), l'eau (el agua). Ces signes linguistiques dont les référents se trouvent dans la réalité environnante traduisent chez Jean de la Croix comme une insuffisance des mots à exprimer l'immensité et la grandeur de l'amour ainsi que la pensée mystique, insuffisance compensée par des similitudes, des comparaisons, des allégories, des analogies certes, mais aussi par le recours à la même configuration littérale du signifiant comme voie d'expression de l'indicible. Au sein d'une syntaxe exclusivement nominale et exclamative, le ressassement chez Jean de la Croix, écrit Nadine $\mathrm{Ly}^{21}$, " opère deux effets de

21 Ly Nadine, La spatialisation du "Je ne sais quoi», in Espaces : séminaire d'études littéraires, PUM, Toulouse, 1988, p. 105. 
dissolution : la sublimation du sens dénoté qui se volatilise au bénéfice de la seule attente du signifiant; la sublimation du signifiant physique lui-même, devenu support de sa propre attente, perspective ouverte sur lui-même, conçu comme projet rythmique, phonique, associé aux mêmes séquences et indéfiniment appelé à se nourrir de sa propre redite inassouvie ". Il en va de même pour le discours poétique de Blas de Otero où le signifiant comme perspective ouverte sur lui-même est toujours en attente de significations et de fonctions nouvelles pour assurer la réalisation du projet poétique.

C'est la " reviviscence " du signifiant qui est placée au cœur du travail poétique. Cette reviviscence ou revitalisation d'un mot, parfois tombé en désuétude, consiste à l'actualiser avec la même signification ou à lui attribuer une signification nouvelle, comme l'explique Manuel Alvar Ezquerra : «En realidad, la revitalización no es un proceso ni de creación ni de incorporación léxica, todo lo más es de renovación semántica, pues la voz ya existía en la lengua. El vocabulario no se ve aumentado cuantitativamente, aunque sí cualitativamente. En relación con el proceso de revitalización léxica podríamos poner algunos casos de desplazamientos semánticos (los cambios categoriales, no), como la motivación del signo lingüístico y las creaciones metafóricas, metonímicas, etc., pues también son procedimientos semánticos para aumentar el caudal de la lengua ${ }^{22}$ Il convient de compléter et d'enrichir cette notion de renouveau sémantique avec celle de renouveau ou d'innovation sémiotique qui contribue, dans le temps et dans l'espace à la réactualisation et à la perpétuation de toutes les qualités phoniques, rythmiques, rimiques, métriques du signifiant dont la primauté a été si chère à Ferdinand de Saussure. À propos de cette pratique sémiotique de l'écriture, Julia Kristeva écrit ceci : " Et qui sait, peut-être une des raisons d'être de la linguistique est-elle de purifier le langage de ces couches de "significations" et d'“interprétations" figées, de concepts a priori et de logique déjà faite $»^{23}$.

Que le signifiant soit linéaire ou non au sein de la chaîne signifiante, il est mis au service de l'efficacité et du dynamisme du travail associatif dans l'écriture poétique de Blas de Otero. Quant à son Cántico espiritual, ce n'est pas la reprise d'une série de termes ressassés par le poète mystique qui vont assurer la qualité poétique de la création du poète moderne, mais bien l'usage et l'objectif personnels dans un renouvellement permanent d'associations et de figures de style, où le yo autobiographique et poétique exprime sa "subjectivité instante et élusive ", selon l'expression d'Émile Benveniste. De nouveaux rapports associatifs s'établissent au sein du texte poétique dont la signification dépasse et transporte la signification première. Cette inspiration originelle et originale de nature lexicale, thématique, esthétique, sert à Blas de Otero pour réaliser sa propre signature poétique, colorée par ses préoccupations pour la condition humaine dans ce $\mathrm{XX}^{\mathrm{e}}$ siècle tiraillé à

22 Alvar Ezquerra Manuel, La formación de palabras en español, Arco Libros, Madrid, 2012, p. 13.

23 Kristeva Julia : Semeiotikè. Recherches pour une sémanalyse, Éditions su Seuil, Paris, 1969, p. 205. 
la fois entre les guerres, les brutalités, les souffrances et le progrès, les avancées sociales, la recherche du bonheur, d'épanouissement, d'idéal.

Il convient de remarquer que Blas de Otero, non seulement reprend le titre Cántico espiritual pour intituler son premier recueil, mais l'incorpore à son texte poétique dans le Cántico II et ensuite pour clôturer le Cántico III :

Cántico de mi vida inusitada (II, p. 102)

Estoy llamando à Ti con este cántico (II, p. 102)

Cántico blanco que te ofrezco a Ti,

Señor de las estrellas condolidas.

Cántico espiritual

Sobre el barro que asienta mi garganta (III, p. 104)

Le signifiant « cántico » est hypogrammatisé dans les vers suivants :

Te CANto a TI COn el amor divino

$\mathrm{Y}$ esTe resCOldo del humANo amor.

Te CANTO a tI, doliéndome de todo,

SubIEndo PoR Tu NOChe hAcIa mI AUrora. (II, p. 101)

Cette strophe de Blas de Otero, où les trois premiers vers anagrammatisent le signifiant " cántico » et le quatrième " cántico espiritua (l) ", mais également les vers de Saint-Jean de la Croix analysés précédemment " al aiRe de Tu vuElO y fREscO Toma ", " PasTORESs, los que fueredes ", "si pOR vEnTura vieredes " qui anagrammatisent les signifiants "otero/Otero", sont des exemples qui obéissent aux lois du langage poétique telles que les a énoncées Ferdinand de Saussure dans ses cahiers d'Anagrammes, où il constate que la plupart des vers contiennent des noms de divinités ou de personnages importants dont les syllabes sont dispersées dans divers signifiants de la chaîne linéaire. En effet, à l'écoute des vers cités, le lecteur « entend s'élever, de proche en proche, les phonèmes principaux d'un nom propre [du poète et du poème], séparés les uns des autres par des éléments phonétiques indifférents $~_{24}$. Ce matériel "prêtà-composer " constitué d'anagrammes et d'hypogrammes renvoie à la notion saussurienne de «sociation psychologique » qui représente le degré d'élaboration à la fois le plus haut et le plus profond au moment où la pensée jaillit du cerveau et se matérialise en écriture. En dehors de sa dimension sociale, cette « sociation psychologique inévitable et profonde » suppose, selon Chloé Laplantine ${ }^{25}$ " une union infiniment subjective, telle que le sujet se constitue lui-même dans et par l'activité de production de la forme qu'il donne à sa pensée ", c'est-à-dire l'exercice de l'inconscient, qualifié par Federico Bravo ${ }^{26}$ d'inconscient psycholinguistique ou encore de procédé/opération neuro-psycholinguistique.

24 Ibid., p. 28.

25 Laplantine Chloé, Le sentiment de la langue, 2005, article disponible sur le site http://www. univ-paris8.fr.

26 Bravo Federico, Anagrammes. Sur une hypothèse de Ferdinand de Saussure, Éditions LambertLucas, Limoges, 2011. 
Bien que la systématicité des lois de la composition énoncées par Saussure ne soit pas toujours au rendez-vous, leur mérite est de mettre au jour cette capacité du langage poétique à occulter des significations supplémentaires que seul le travail sur le signifiant permet de percer sous l'écorce du message linéaire pour en trouver un autre sous-jacent, superposé, inconscient. Il est à noter que le diphone central du mot-thème " cántico " TI renvoie à la deuxième personne d'interlocution, participant ainsi de l'instauration du dialogue inter et intrapoétique dont l'efficacité et la solidité sont renforcées par les " frontières " du signifiant Cántic $\mathbf{O}$ posées par le « $\mathbf{C}$ » de « $\mathbf{C r u z}$ » et par le « $\mathbf{O}$ » de " Otero ».

Quant, au chiasme " amor divino/humano amor " figurant dans les vers "Te canto a Ti con el amor divino/y este rescoldo del humano amor ", il insiste sur le fait que le "cántico » est un hymne d’amour dédié à Dieu, mais aussi au poète mystique, et de manière générale à l'être humain, ce qui marque une première rupture entre les deux discours poétiques. Si les deux recueils se prêtent parfaitement à une lecture à la fois religieuse et mystique, profane et érotique et si dans la poésie de Jean de la Croix, les allégories de l'amour divin se trouvent incarnées par les signifiants Amado/Amada, Esposo/Esposa, paloma/ ciervo, dans la poésie de Blas de Otero, l'amour divin et l'amour humain vont de pair dès le vers apertural du Cántico espiritual: "Todo el amor divino, con el amor humano ". Cette symbiose de l'amour divin et de l'amour humain se densifie avec l'apparition dans les vers suivants des signifiants "mujer", " cuerpo ", « hombre ": « tú mujer que te enciendes y te apagas» (p. 99); " mujer pura " (p. 99) ; « la unidad de mi alma y de mi cuerpo » (p. 99); « mi cuerpo desmorrona a cada instante " (p. 100); " oh carne de mi carne y de mi alma " (p. 100) ; " de mi cuerpo en llanto " (p. 100) ; " mi pequeñez de hombre " (p. 108). Cette posture humanisante endossée par le signifiant va participer par la suite de l'humanisation de la lettre, du mot, du vers, de la poésie et de l'écriture tout entière de Blas de Otero.

La dimension divine, générale, universelle de Jean de la Croix devient, par le procédé poétique du rapprochement, humaine, singulière, particulière chez Blas de Otero. Le rapprochement et la superposition du divin et de l'humain accompagnent la singularisation du yo poétique. La vie qu'insuffle le poète au signifiant pour le rendre plus palpable et plus puissant, sert de transition du divin, du général et de l'universel à l'humain, au particulier et au singulier, ayant justement pour but de suspendre l'universel « cerrándome el oído/al gozne del Universo suspendido", (p. 116). La suspension de l'universel permet d'identifier un espace plus concret et plus précis qui coïncide avec le pays du poète : «el cielo castellano " (p. 112), " esa ola amarilla del campo de Castilla » (p. 117); "Que si ella es castellana/de dios, lo que del mundo yo más quiero " (p. 113).

La dimension humaine du signifiant, cette "palabra fieramente humana", comme aurait pu le dire le poète lui-même, lui permet de mettre à nu le code poétique classique et de nouer une infinité de liens associatifs poétiques. 
L'humanisation du mot par l'écriture face à la déshumanisation du monde par les guerres et par les dictatures permettra à Blas de Otero d'exprimer ses pensées les plus profondes et de s'accomplir dans sa destinée de poète.

À la lumière des vers «Aún estoy más abajo de los hombres, /en esta sepultura de comienzos " (p. 101), le premier recueil de Blas de Otero, Cántico espiritual, représente le commencement d'une production poétique féconde qui permettra au poète de tendre vers la vie éternelle par la grâce de Dieu, mais également par la grâce de la poésie, comme l'écrit le poète lui-même dans les vers qui suivent :

Oh eterna poesía.

Eterna como el hombre que la ha hecho.

el cuerpo se moría...,

nacíanle del pecho

Alas de eternidad, sobre su techo. (p. 110)

"Les ailes d'éternité " de Blas de Otero qui ont connu leurs premiers battements dans la sublime poésie de Jean de la Croix, lui ont permis de s'envoler vers les hauteurs du " otero » de la poésie espagnole, grâce à la poétisation du signifiant et à l'exploration des automatismes associatifs poétiques, les deux se trouvant au cœur de son écriture.

\title{
Violeta Bereghici \\ EA3656 AMERIBER
}

Université Bordeaux Montaigne violeta. bereghici@u-bordeaux-montaigne.fr

\begin{abstract}
Résumé
Cette étude comparative de deux recueils ayant le même titre - Cántico espiritual-, écrits à un intervalle de quatre siècles par deux poètes majeurs de la poésie espagnole - Jean de la Croix et Blas de Otero -, a pour objectif de montrer que le signifiant, par sa résistance et par sa prégnance, par son autonomie et par sa liberté, est apte à s'ériger en automatisme associatif poétique comme source inépuisable de significations nouvelles.
\end{abstract}

\section{Mots-clés}

Jean de la Croix, Blas de Otero, automatisme associatif poétique, poésie, signifiant, littéralité, résistance.

\begin{abstract}
This comparative study of two anthologies which share the same title - Cántico espiritual written four centuries apart by two major authors of Spanish poetry - Jean de la Croix and Blas de Otero - aims to show how the signifier, with its resistance and weight, with its autonomy and freedom, can work as a poetical associative automatism and become an unending source of new meanings.
\end{abstract}

\section{Keywords}

Jean de la Croix, Blas de Otero, poetical associative automatism, poetry, signifier, literality, resistance. 